\title{
RESEARCH
}

Open Access

\section{Positive change in asthma control using therapeutic patient education in severe uncontrolled asthma: a one-year prospective study}

Xiaoxian Zhang ${ }^{1 \dagger}$, Zhengdao Lai ${ }^{2 \dagger}$, Rihuang $\mathrm{Qiu}^{3}, \mathrm{E} \mathrm{Guo}{ }^{4}$, Jing $\mathrm{Li}^{1}$, Qingling Zhang ${ }^{1}$ and Naijian $\mathrm{Li}^{1^{*}}$

\begin{abstract}
Background: Severe asthma is difficult to control. Therapeutic patient education enables patients to better understand their disease and cope with treatment, but the effect of therapeutic patient education in severe uncontrolled asthma is unclear. We evaluated whether therapeutic patient education is effective in improving asthma control and decreasing the frequency of exacerbations in severe uncontrolled asthma.
\end{abstract}

Methods: This was a prospective, observational, and self-controlled study that enrolled 40 subjects with severe uncontrolled asthma. Patients were seen at a clinic four times (on day 1 and after 3, 6, and 12 months). After baseline data collection, the subjects completed a therapeutic patient education program and were also followedup via telephone after 1, 2, 4, 5, 7, 8, 9, 10, and 11 months to monitor asthma medication adherence and collect asthma-related information.

Results: Within the 1-year study period, a total of 23 exacerbations were recorded in 14 patients, seven of whom required emergency treatment and two of whom were hospitalized. Twelve months after the standardized therapeutic patient education program, pulmonary function and fractional exhaled nitric oxide levels improved significantly in all 40 patients. Moreover, the scores from three standardized asthma questionnaires and indices suggested improved quality of life in these patients with severe uncontrolled asthma. Serum levels of biomarkers reflecting asthma immune responses did not change between baseline and the 1-year follow-up time point.

Conclusions: Therapeutic patient education is effective in improving asthma control and decreasing exacerbations in patients with severe uncontrolled asthma.

Keywords: Severe uncontrolled asthma, Therapeutic patient education, Asthma control

\footnotetext{
* Correspondence: naijian@126.com

${ }^{+}$Xiaoxian Zhang and Zhengdao Lai contributed equally to the work.

'Department of Allergy and Clinical Immunology, State Key Laboratory of Respiratory Disease, National Clinical Research Center for Respiratory Disease, Guangzhou Institute of Respiratory Health, the First Affiliated Hospital of

Guangzhou Medical University, 151 Yan Jiang Rd, 510000 Guangzhou, P.R.

China

Full list of author information is available at the end of the article
}

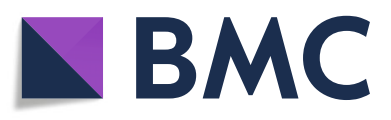

(-) The Author(s). 2021 Open Access This article is licensed under a Creative Commons Attribution 4.0 International License, which permits use, sharing, adaptation, distribution and reproduction in any medium or format, as long as you give appropriate credit to the original author(s) and the source, provide a link to the Creative Commons licence, and indicate if changes were made. The images or other third party material in this article are included in the article's Creative Commons licence, unless indicated otherwise in a credit line to the material. If material is not included in the article's Creative Commons licence and your intended use is not permitted by statutory regulation or exceeds the permitted use, you will need to obtain permission directly from the copyright holder. To view a copy of this licence, visit http://creativecommons.org/licenses/by/4.0/. The Creative Commons Public Domain Dedication waiver (http://creativecommons.org/publicdomain/zero/1.0/) applies to the data made available in this article, unless otherwise stated in a credit line to the data. 


\section{Background}

Asthma is a worldwide problem that currently affects an estimated 358 million individuals [1]. Over the past decade, the aims of asthma management have altered to focus on achieving and maintaining good asthma control and reducing future risks, such as decreases in lung function, asthma exacerbations, hospitalizations, adverse effects from treatment, and death [2]. Despite the widespread dissemination of asthma management guidelines, many patients have inadequately controlled disease and experience frequent asthma exacerbations [3, 4]. Patients who experience symptoms and severe asthma attacks even with high-dose inhaled corticosteroids plus another controller medication and/or systemic corticosteroids are defined as having severe uncontrolled asthma $[3,5]$. Between 2.3 and $3.6 \%$ of patients with persistent asthma have severe uncontrolled asthma [6], but this minority accounts for substantially greater asthma-related healthcare resource use and costs than the majority [7].

Treatment strategies for severe uncontrolled asthma have focused on the use of anti-inflammatory and bronchodilator drugs [3]. Despite multiple treatment options, therapy for severe uncontrolled asthma remains a challenge. Asthma control is affected by multiple factors, which also require prevention measures to decrease negative behaviors and aggravating factors $[8,9]$. Previous studies provide evidence for the efficacy and efficiency of patient education and asthma self-management $[10,11]$; therapeutic patient education, which helps patients acquire or maintain the skills they need to self-manage a chronic disease [12], has been well-studied in asthma control in children [13]. However, whether therapeutic patient education is effective in improving asthma control in severe uncontrolled asthma cases is unknown. The aim of this study was to investigate the effect of therapeutic patient education on asthma control, exacerbations, pulmonary function, quality of life, and serum biomarkers in patients with severe uncontrolled asthma.

\section{Methods \\ Study design}

We conducted a prospective single-center study in the Department of Allergy and Clinical Immunology, the First Affiliated Hospital of Guangzhou Medical University (Guangzhou, China). All patients provided written informed consent and the study was approved by the Chinese Clinical Trial Registry (ChiCTR-TRC12,002,527). A total of 40 subjects with severe uncontrolled asthma were included in the study, to be seen at the clinic four times over 12 months. On visit 1 (day 1 ), the subjects were screened and baseline data were collected, including demographic characteristics, medical history, serum biomarker levels, asthmaspecific assessment, asthma-related healthcare use, and an asthma assessment questionnaire. After baseline data collection, the subjects then completed therapeutic patient education. All subjects were required to attend three follow-up visits at 3 (visit 2), 6 (visit 3), and 12 (visit 4) months. All subjects were also contacted by telephone after 1, 2, 4, 5, 7, 8, 9, 10, and 11 months to monitor asthma medication adherence and collect asthma-related information. Table 1 details the study design.

\section{Patients}

Patients with severe uncontrolled asthma were defined as subjects whose asthma was inadequately controlled, as assessed by the five-item Asthma Control Questionnaire (ACQ-5) score of $\geq 1.5$ with one or more uncontrolled asthma symptoms consistent with the Global Initiative for Asthma guidelines (nighttime awakening $\geq 1$ time/week, symptoms $>2$ days/week, shortacting beta-agonist use $>2$ days/week, or interference

Table 1 Schedule of assessments

\begin{tabular}{|c|c|c|c|c|c|}
\hline \multirow[t]{3}{*}{ Assessment/procedure } & \multicolumn{5}{|l|}{ visit } \\
\hline & 1 & 2 & 3 & 4 & Telephone visit \\
\hline & Day 1 & 3 months after Day 1 & 6 months after Day 1 & 12 months after Day 1 & $\begin{array}{l}1,2,4,5,7,8,9,10 \text { and } \\
11 \text { months after Day } 1\end{array}$ \\
\hline Medical history & $\checkmark$ & & & & \\
\hline TPE & $\checkmark$ & $\checkmark$ & $\checkmark$ & $\checkmark$ & \\
\hline Spirometry & $\checkmark$ & & $\checkmark$ & $\checkmark$ & \\
\hline Exacerbation assessment & $\checkmark$ & $\checkmark$ & $\checkmark$ & $\checkmark$ & $\checkmark$ \\
\hline PRO & $\checkmark$ & $\checkmark$ & $\checkmark$ & $\checkmark$ & \\
\hline FeNO & $\checkmark$ & & $\checkmark$ & $\checkmark$ & \\
\hline Biomarker test & $\checkmark$ & & $\checkmark$ & $\checkmark$ & \\
\hline
\end{tabular}

TPE therapeutic patient education; PRO patient report outcomes (Asthma Control Questionnaire 5, Standardized Asthma Quality of Life Questionnaire, and Asthma Symptom Utility Index); FeNO fractional exhaled nitric oxide 
with daily activities) despite daily use of inhaled corticosteroid corresponding to $500-2,000 \mu \mathrm{g} /$ day of fluticasone propionate dry powder inhaler or the equivalent and a second eligible asthma controller medication [2].

Subjects were required to meet the following criteria for study entry:

1. Aged $18-75$ years and had been diagnosed with asthma for at least 1 year.

2. Pre-bronchodilator forced expiratory volume in $1 \mathrm{~s}$ $\left(\mathrm{FEV}_{1}\right)$ of $40-80 \%$ of predicted value at visit 1.

3. Bronchodilator response $\geq 12 \% \mathrm{FEV}_{1}$ improvement in response to $400 \mu \mathrm{g}$ of an inhaled short-acting beta-agonist at visit 1 .

4. Using an eligible second controller medication (long-acting beta 2-agonist, long-acting muscarinic antagonist, leukotriene receptor antagonist, or theophylline) within prescribed dosing range for $\geq 6$ months prior to visit 1 with no anticipated changes throughout the study.

Subjects who met any of the following criteria prior to enrolment were excluded from the study:

1. Received maintenance oral corticosteroid therapy, defined as daily or alternate day oral corticosteroid maintenance therapy within the preceding 3 months.

2. Treated with systemic (oral, intravenous, or intramuscular) corticosteroids within the preceding 4 weeks.

3. Having a history of cystic fibrosis, chronic obstructive pulmonary disease, or other clinically significant lung disease other than asthma.

4. Being a current smoker or former smoker with a smoking history of $>10$ pack-years.

\section{Intervention}

A five-item therapeutic patient education course was created to address learning objectives for asthma control according to the recommendations of the National Standards for Asthma Self-Management Education and the Global Strategy for Asthma Management and Prevention [2, 14]. Patients participated in an initial individual therapeutic patient education course on visit 1 after baseline data collection. The course was provided by a team consisting of an asthma specialist nurse and asthma physician experienced in therapeutic patient education. The therapeutic patient education intervention addressed asthma pathophysiology, illness perceptions, medication skills, self-monitoring techniques, and environmental control and avoidance strategies (reducing exposure). All subjects were required to repeat the therapeutic patient education course in person at the subsequent three visits to the clinic (after 3, 6, and 12 months). Detailed therapeutic patient education course information can be obtained by scanning the WeChat public account link in the supplementary material.

\section{Atopy}

All subjects underwent an epicutaneous prick allergy test for 11 allergen extracts (ALK-Abelló, Hørsholm, Denmark) in the volar surface of the forearm.

\section{Outcome measures \\ Asthma control}

The primary outcome of asthma control was measured by the ACQ-5, Standardized Asthma Quality of Life Questionnaire (AQLQ(S)), and Asthma Symptom Utility Index (ASUI) [15-17]. These short screening instruments have been developed for use in everyday clinical practice, to identify patients with poor asthma control without measuring lung function. For this study, an asthma exacerbation was defined as new or increased asthma symptoms (including wheezing, cough, dyspnea, chest tightness, and nocturnal awakenings because of these symptoms) that led to treatment with systemic corticosteroids or to hospitalization. Treatment with systemic corticosteroids was defined as treatment with oral, intravenous, or intramuscular corticosteroids for at least 3 days or an emergency department visit with at least one intravenous or intramuscular dose of corticosteroids. Medication adherence was calculated as the percentage of prescribed doses taken each week [18].

\section{Pulmonary function, fractional exhaled nitric oxide (FeNO) measurement, and serum biomarkers}

Subjects completed a pulmonary function test and FeNO measurement at baseline and after 6 and 12 months. Prior to spirometry, airway inflammation was assessed by the FeNO test using a NIOX Flex device (Aerocrine, Solna, Sweden) in accordance with international guidelines [19]. Values are expressed as parts per billion, with a normal FeNO range for healthy adults defined as $5-25 \mathrm{ppb}$. Spirometry data were obtained by trained respiratory therapists using a portable spirometer (MasterScreen PNEUMO, CareFusion, San Diego, CA, USA) [20]. Serum cytokine levels were assayed using Luminex xMap (Luminex, Austin, TX, USA) and a commercially available cytokine 12-plex panel (Bio-Rad, Hercules, CA, USA) according to the manufacturers' guidelines and measured on a Bio-Plex 200 platform (Bio-Rad). 


\section{Statistical analysis}

We analyzed the data using paired tests comparing asthma control (asthma exacerbation, pulmonary function, and FeNO), quality of life (standardized questionnaire scores), and serum biomarker levels at baseline and 12 months after the initial therapeutic patient education. A paired Student's $t$-test (or nonparametric Wilcoxon signed-rank test when necessary) was used to compare quantitative variables. Statistical analysis was performed in SPSS version 24 (IBM SPSS, Armonk, NY, USA), and $p<0.05$ was considered significant.

\section{Results}

\section{Population}

A total of 40 subjects with severe uncontrolled asthma (25 women and 15 men) were included in the study. Mean age was 50.3 years (standard deviation: 11.3). Median duration of asthma diagnosis was 10.5 years, with an interquartile range of 5.0-19.5. Thirty of the 40 patients tested positive for one or several relevant allergens on the skin prick test. Eleven patients had a personal history of atopic dermatitis, 28 suffered from rhinitis, and 16 from conjunctivitis. Table 2 lists patient characteristics at baseline.

\section{Asthma exacerbation and medication adherence}

During the 1-year study period, a total of 23 exacerbations had been recorded in 14 patients, seven of whom required emergency treatment and two of whom required hospitalization. The average number of exacerbations was 0.58 per person, and each

Table 2 Characteristics of study patients

\begin{tabular}{ll}
\hline Characteristics & Total $(\boldsymbol{n}=\mathbf{4 0})$ \\
\hline Age (y) & $50.3 \pm 11.3$ \\
Gender (female/male) & $25 / 15$ \\
Body mass index $\left(\mathrm{kg} / \mathrm{m}^{2}\right.$ ) & $24.1 \pm 4.4$ \\
Smoking status (never/ex), $\mathrm{n}$ & $40 / 0$ \\
Atopy, $\mathrm{n}(\%)$ & $30(75.0 \%)$ \\
Duration of asthma (y) & $10.5(5.0-19.5)$ \\
Combine with rhinitis & $28(70.0 \%)$ \\
Combine with atopic dermatitis & $11(27.5 \%)$ \\
Combine with conjunctivitis & $16(40.0 \%)$ \\
Dose of inhaled corticosteroids (ug/d) & $1000(1000-2000)$ \\
Budesonide/formoterol, $\mathrm{n}(\%)$ & $24(60 \%)$ \\
Fluticasone/salmeterol, $\mathrm{n}(\%)$ & $16(40 \%)$ \\
Montelukast Sodium ${ }^{\mathrm{a}}, \mathrm{n}(\%)$ & $28(70 \%)$ \\
Eos in blood(x10 $\left.{ }^{3} / \mathrm{uL}\right)$ & $0.27 \pm 0.20$ \\
\hline
\end{tabular}

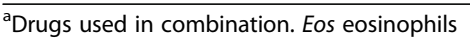

exacerbation lasted an average of 8.4 days. Asthma exacerbations during the study period decreased significantly from the number of exacerbations reported for the preceding 1-year period $(2.80 ; p<0.001$; Fig. 1 A). Mean ( \pm standard deviation) medication adherence for all patients at visits 1,3 , and 4 was $75.5 \% \pm 6.7 \%, 83.6 \% \pm 7.2 \%$, and $90.5 \% \pm 6.0 \%$, respectively, indicating improved adherence over time $(p<0.01$; Fig. 1B).

\section{Pulmonary function and FeNO}

Over the course of the study, pulmonary function improved and airway inflammation decreased significantly in the 40 patients. Predicted $\mathrm{FEV}_{1}$ increased from $59.2 \%$ $\pm 1.8 \%$ at baseline to $66.6 \% \pm 2.9 \%$ after 6 months ( $p=$ $0.01)$ and to $66.6 \% \pm 3.0 \%(p=0.011)$ after 12 months (Fig. 1 C). Similarly, FeNO levels decreased from $36.0 \pm$ $5.6 \mathrm{ppb}$ at baseline to $24.7 \pm 3.2 \mathrm{ppb}(p=0.023)$ after 6 months and to $23.7 \pm 3.6 \mathrm{ppb}(p=0.001)$ after 12 months (Fig. 1D).

\section{Quality of life}

Six months after beginning the study, the questionnaire scores of the 40 patients (ACQ-5, AQLQ(S), and ASUI) indicated an improvement of perceived quality of life. ACQ-5 scores decreased significantly between visit $1 \quad(1.9 \pm 0.2)$ and visit $4 \quad(1.3 \pm 0.7$; Fig. 2 A). ASUI scores increased from visit $1(0.75 \pm$ $0.16)$ to visit $3(0.80 \pm 0.17, p=0.132)$ and visit 4 $(0.85 \pm 0.17, p=0.008$; Fig. 2B). Total AQLQ(S) scores increased during the study period ( $p<0.01$; Fig. 2 C), and in particular the activity domain scores and emotional domain scores (Fig. 2D).

\section{Serum biomarkers}

Levels of interleukins, tumor necrosis factor $\alpha$, eotaxin, and transforming growth factor $\beta 1$ did not change significantly from baseline levels over the study period (Fig. 3).

\section{Discussion}

Therapeutic patient education is a nonpharmacological intervention that assists patients and their families in learning or maintaining relevant skills for managing life with a chronic disease [12, 13, 21], and has been well-studied in asthma, chronic hand eczema, and chronic heart failure [12, 13, 21]. Previous research supports the hypothesis that therapeutic patient education is essential to improving the knowledge of the asthma patient, self-management of the disease, and the prevention of attacks [13]. However, evidence for the efficacy of therapeutic patient education in severe uncontrolled asthma is lacking. We found that therapeutic patient education was effective 

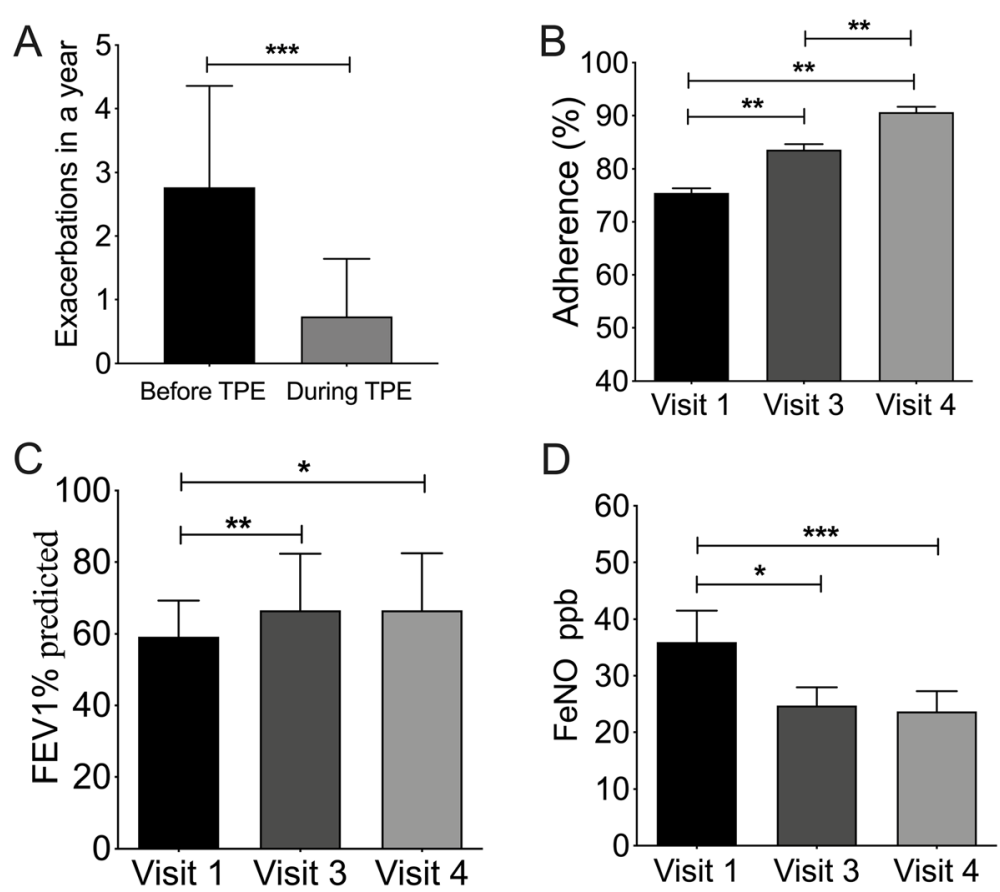

Fig. 1 Changes in participant total exacerbations, spirometry, and fractional exhaled nitric oxide (FeNO) over the course of the study. A Asthma exacerbations decreased significantly after therapeutic patient education (TPE). B-D Measurements were conducted on day 1 (visit 1) and after 6 months (visit 3) and 12 months (visit 4). Predicted forced expiratory volume in $1 \mathrm{~s}\left(\mathrm{FEV}_{1}\right)$ increased significantly and FeNO decreased over time. Medication adherence was calculated as the percentage of prescribed doses taken each week. ${ }^{*} p<0.05,{ }^{* *} p<0.01,{ }^{* * *} p<0.001$

in improving asthma control, decreasing exacerbations, and reducing activity limitations in patients with severe uncontrolled asthma. Pulmonary function improved significantly and airway inflammation was reduced in the study cohort, and questionnaire scores indicated improvement in quality of life.

International guidelines highlight that asthma control (defined as prevention of chronic symptoms, minimal use of rescue medication, no exacerbations, no emergency care, and maintenance of normal levels of physical activity) is the major goal in the long-term management of asthma and suggest that the level of control should be monitored by both health care professionals and patients themselves at regular intervals $[2,3]$. Despite the many available options for managing uncontrolled asthma, a large proportion of asthma patients still experience uncontrolled disease [22]. Numerous factors contribute to uncontrolled asthma, most commonly are poor adherence, poor inhaler technique, and failure to assess control adequately. A review of 24 survey studies revealed that, in general, adolescents and adults with asthma lack knowledge about the underlying causes of asthma symptoms, such as allergies and triggers; have limited knowledge of treatment options, including correct use of medication; and have low expectations of receiving appropriate therapy or of having a positive encounter with their health care professional [23]. Patients with severe uncontrolled asthma who display poor medication adherence may have an increased risk of experiencing poor asthma outcomes [24], and therapeutic patient education can improve disease control in these patients.

Educational interventions covering important aspects of asthma management are required for adequate selfmanagement [25]. Such interventions for children with asthma and their families have been shown to reduce the number of emergency department visits and hospital admissions [26], and may increase medication adherence and consequently reduce the severity and frequency of symptoms, nocturnal awakenings, and activity limitations [27]. Our results show that therapeutic patient education is effective in improving asthma control, decreasing exacerbations, and reducing activity limitations in patients with severe uncontrolled asthma. Notably, we did not detect significant changes in serum levels of asthma biomarkers over time. The non-invasive asthma control indicators (questionnaires, $\mathrm{FEV}_{1}$, and $\mathrm{FeNO}$ ) were more sensitive and convenient for assessing asthma improvement.

The primary limitations of this study are the small sample and the lack of a control group. Without a control group, it is difficult to assess the direct impact of 

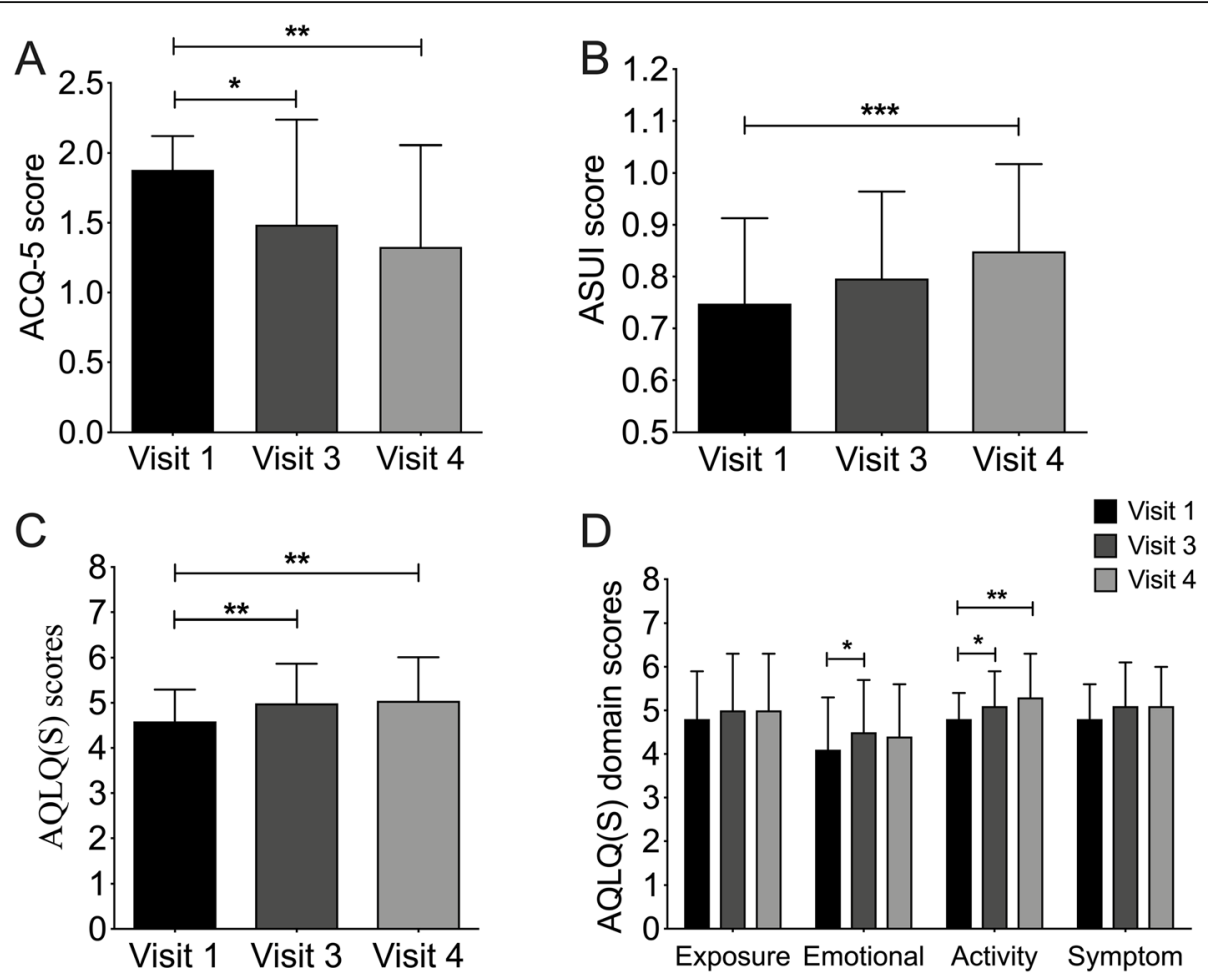

Fig. 2 Asthma symptoms, level of asthma control, and asthma-related quality of life. A Asthma Control Questionnaire 5 scores decreased significantly from visit 1 (day 1) to visit 3 (6 months after baseline) and visit 4 (12 months after baseline). Asthma Symptom Utility Index scores (B) and Standardized Asthma Quality of Life Questionnaire scores $(\mathbf{C})$ increased over time. D Standardized Asthma Quality of Life Questionnaire scores in four domains increased over time, especially the activity domain score after 6 and 12 months and the emotional domain score after 6 months. ${ }^{*} p<0.05,{ }^{* *} p<0.01,{ }^{* * *} p<0.001$

the therapeutic patient education itself on asthma control, quality of life, and asthma exacerbations, as controller medication in addition to the therapeutic patient education would improve these outcomes.

In summary, our standardized therapeutic patient education program appears to be an effective tool for improving asthma control, decreasing exacerbations, and improve activity limitations in patients with severe uncontrolled asthma, but this approach requires a collaborative effort by patients and physicians.

\section{Conclusions}

Therapeutic patient education can be an effective and collaborative tool for improving asthma control and decreasing exacerbations in patients with severe uncontrolled asthma.

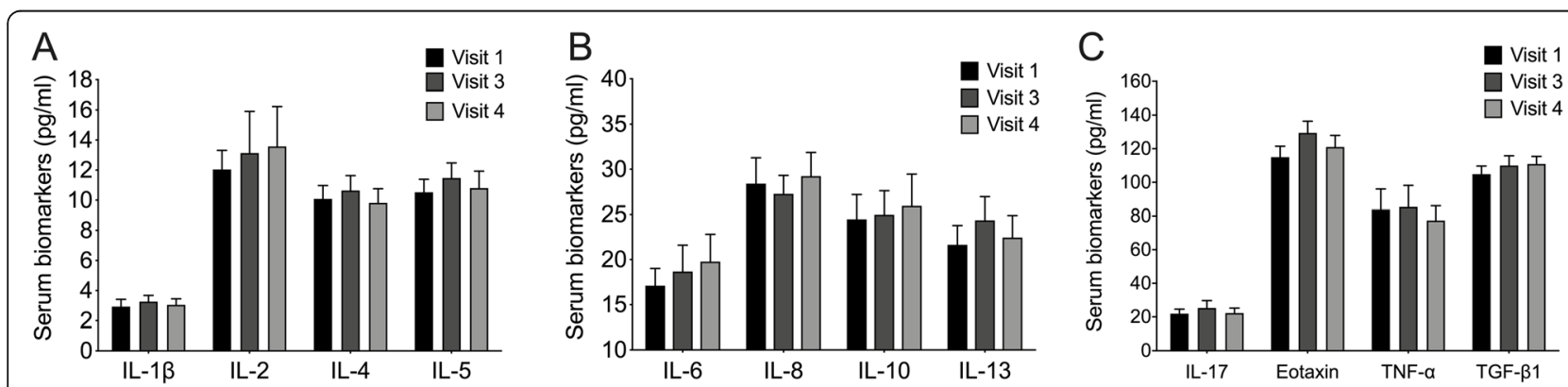

Fig. 3 Changes in serum levels of asthma-related biomarkers. There were no significant changes over time between serum levels of interleukins (IL), tumor necrosis factor a (TNF-a), eotaxin, or transforming growth factor $\beta 1$ (TGF- $\beta 1$ ). Visit 1, day 1; visit 3, 6 months after baseline; visit 4, 12 months after baseline 


\section{Abbreviations}

ACQ-5: Asthma Control Questionnaire 5; AQLQ(S): Standardized asthma quality of life questionnaire; ASUI: Asthma symptom utility index; FEV1: Forced expiratory volume in $1 \mathrm{~s}$; FeNO: Fractional exhaled nitric oxide; TPE: Therapeutic patient education

\section{Supplementary Information}

The online version contains supplementary material available at https://doi. org/10.1186/s40733-021-00076-y.

\section{Additional file 1.}

\section{Acknowledgements}

We thank Liwen Bianji (Edanz) (www.liwenbianji.cn), for editing the English text of a draft of this manuscript.

\section{Authors' contributions}

$N J L, Q L Z$ and $J L$ conceived the study, directed the project, and designed the experiments; XXZ, ZDL, RHQ and EG obtained the samples, interpreted the results, and analyzed the data; ZDL and XXZ performed the data analysis and wrote the manuscript; all authors read and approved the final manuscript.

\section{Funding}

This work was supported by a grant of the Precision Medicine Research of The National Key Research and Development Plan of China (2016YFC0905800), Science and Technology Program of Guangdong province (2017B020226006), National Natural Science Foundation of China (91542104).

\section{Availability of data and materials}

Data is available upon reasonable request.

\section{Declarations}

\section{Ethics approval and consent to participate}

The Ethics Commission of the First Affiliated Hospital of Guangzhou Medical University approved the study (No.2012-8). Volunteers provided voluntary, written informed consent before taking part in study procedures. Trial registration: ChiCTR-TRC-12002527, registered 20 September 2012 retrospectively (http://www.chictr.org.cn/showproj.aspx?proj=7024).

\section{Consent for publication}

Not applicable.

\section{Competing interests}

There are no conflicts of interest to declare. There is also no non-author involvement in the preparation of the manuscript.

\section{Author details}

${ }^{1}$ Department of Allergy and Clinical Immunology, State Key Laboratory of Respiratory Disease, National Clinical Research Center for Respiratory Disease, Guangzhou Institute of Respiratory Health, the First Affiliated Hospital of Guangzhou Medical University, 151 Yan Jiang Rd, 510000 Guangzhou, P.R. China. ${ }^{2}$ Department of Pulmonary and Critical Care Medicine, Dongguan People's Hospital, 523000 Dongguan, P.R. China. ${ }^{3}$ Department of Respiratory Medicine, The Affiliated Ganzhou Hospital of Nanchang University, 341000 Ganzhou, P.R. China. ${ }^{4}$ Xiangyang Central Hospital, Hubei University of Arts and Science, 441000 Xiangyang, P.R. China.

Received: 12 April 2021 Accepted: 3 July 2021

Published online: 21 July 2021

\section{References}

1. GBD 2015 Chronic Respiratory Disease Collaborators. Global, regional, and national deaths, prevalence, disability-adjusted life years, and years lived with disability for chronic obstructive pulmonary disease and asthma, 19902015: a systematic analysis for the Global Burden of Disease Study 2015. Lancet Respir Med. 2017;5(9):691-706.

2. Global Initiative for Asthma. Global Strategy for Asthma Management and Prevention, 2014. Available from: http://www.ginasthma.org/.
3. Chung KF, Wenzel SE, Brozek JL, et al. Teague WG: International ERS/ATS guidelines on definition, evaluation and treatment of severe asthma. Eur Respir J. 2014:43:343-73.

4. Mincheva $R$, Ekerljung $L$, Bossios $A$, et al. High prevalence of severe asthma in a large random population study. J Allergy Clin Immunol. 2018;141(6):2256-64.e2.

5. Israel E, Reddel HK. Severe and Difficult-to-Treat Asthma in Adults. N Engl J Med. 2017;377(10):965-76.

6. Hekking PP, Wener RR, Amelink $M$, et al. The prevalence of severe refractory asthma. J Allergy Clin Immunol. 2015;135(4):896-902.

7. Kerkhof M, Tran TN, Soriano JB, et al. Healthcare resource use and costs of severe, uncontrolled eosinophilic asthma in the UK general population. Thorax. 2018;73(2):116-24.

8. Cardet JC, Louisias M, King TS, et al. Income is an independent risk factor for worse asthma outcomes. J Allergy Clin Immunol. 2018;141(2):754-60.e3.

9. Huang $K$, Yang T, Xu J, China Pulmonary Health (CPH) Study Group. Prevalence, risk factors, and management of asthma in China: a national cross-sectional study. Lancet. 2019;394(10196):407-18.

10. Boulet LP. Asthma education: an essential component in asthma management. Eur Respir J. 2015:46(5):1262-4.

11. Hsu J, Wilhelm N, Lewis $L$, et al. Economic Evidence for US Asthma SelfManagement Education and Home-Based Interventions. J Allergy Clin Immunol Pract. 2016:4(6):1123-34.e27.

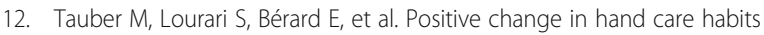
using therapeutic patient education in chronic hand eczema. Contact Dermatitis. 2020;82(1):10-7.

13. Martínez-González CL, Camargo-Fajardo MCC, Segura-Medina P, et al. Therapeutic Patient Education with Learning Objects Improves Asthma Control in Mexican Children. J Med Syst. 2020;44(4):79.

14. Gardner A, Kaplan B, Association of Asthma Educators, et al. National standards for asthma self-management education. Ann Allergy Asthma Immunol. 2015;114(3):178-186.e1.

15. Juniper EF, O'Byrne PM, Guyatt GH, et al. Development and validation of a questionnaire to measure asthma control. Eur Respir J. 1999;14(4):902-7.

16. Juniper EF, Buist AS, Cox FM, et al. Validation of a standardized version of the Asthma Quality of Life Questionnaire. Chest. 1999;115(5):1265-70.

17. Revicki DA, Leidy NK, Brennan-Diemer F, et al. Integrating patient preferences into health outcomes assessment: the multiattribute Asthma Symptom Utility Index. Chest. 1998;114(4):99-107.

18. Janson SL, McGrath KW, Covington JK, et al. Individualized asthma selfmanagement improves medication adherence and markers of asthma control. J Allergy Clin Immunol. 2009;123(4):840-6.

19. American Thoracic Society, European Respiratory Society. ATS/ERS recommendations for standardized procedures for the online and offline measurement of exhaled lower respiratory nitric oxide and nasal nitric oxide, 2005. Am J Respir Crit Care Med. 2005;171:912-30.

20. Liu S, Zhou Y, Liu S, et al. Association between exposure to ambient particulate matter and chronic obstructive pulmonary disease: results from a cross-sectional study in China. Thorax. 2016;0:1-8.

21. Juillière $Y$, Jourdain P, Suty-Selton C, ODIN Cohort Participants. Therapeutic patient education and all-cause mortality in patients with chronic heart failure: a propensity analysis. Int J Cardiol. 2013;168(1):388-95.

22. Sullivan SD, Rasouliyan L, Russo PA, et al. Extent, patterns, and burden of uncontrolled disease in severe or difficult-to-treat asthma. Allergy. 2007;62(2):126-33.

23. Holgate ST, Price D, Valovirta E. Asthma out of control? A structured review of recent patient surveys. BMC Pulm Med. 2006;(6)(suppl 1):S2.

24. Sulaiman I, Greene G, MacHale E, et al. A randomised clinical trial of feedback on inhaler adherence and technique in patients with severe uncontrolled asthma. Eur Respir J.2018;51(1):1701126.

25. Al-Jahdali $\mathrm{H}$, Ahmed A, Al-Harbi A, et al. Improper inhaler technique is associated with poor asthma control and frequent emergency department visits. Allergy Asthma Clinical Immunology. 2013:9(1):8.

26. Watson WT, Gillespie C, Thomas N, et al. Small-group, interactive education and the effect on asthma control by children and their families. CMAJ. 2009:181(5):257-63.

27. Morisky DE, Kominski GF, Afifi AA, et al. The effects of a disease management program on self-reported health behaviors and health outcomes: evidence from the "Florida: a healthy state (FAHS)" Medicaid program. Health Educ Behav. 2009;36:505-17.

\section{Publisher's Note}

Springer Nature remains neutral with regard to jurisdictional claims in published maps and institutional affiliations. 